\title{
Gender determination in adults using calcaneal diameters from lateral foot X-ray images in the Iranian population
}

\author{
Foroozan Faress ${ }^{1}$, Maryam Ameri ${ }^{1}{ }^{*} \mathbb{D}$, Hanieh Azizi ${ }^{1}$, Hanieh Saboori Shekofte ${ }^{2}$, Rozita Hosseini ${ }^{3}$ \\ Received: 17 Sep 2020 \\ Published: 14 Jun 2021
}

\section{Abstract}

Background: Using morphologic features of the bones is the basis of gender determination in anthropology and forensic medicine. In this study, we evaluated the calcaneus diameters for gender determination in the Iranian population.

Methods: This cross-sectional study was conducted on Iranians referring to Hazrat-e Rasool Hospital's radiology ward for plain lateral X-ray of the foot. Lateral foot X-rays from 100 men and 100 women were gathered and evaluated for calcaneal indexes. These patients aged between 18 and 80 years old who did not suffer major trauma to the calcaneus bone were recruited. Using a picture archiving and communication system (PACS), four variables were calculated for each X-ray graph: Maximum anterior-posterior length of the calcaneus (MAXL), Maximum height of the bone (MAXH), Height of the cuboid facet (CFH) and Height the calcaneal body (BH). Cut off points for each index were calculated using the area under curve (AUC) in ROC curves.

Results: Lateral foot X-rays from 100 men and 100 women were gathered and evaluated for calcaneal indexes. The means of the four indexes were compared between the sexes which showed all four means are significantly different $\mathrm{p}$ value $<0.001$. AUC for MAXL in differentiating the genders was 0.824 , which showed 86.8 as the cut off with a sensitivity of $80 \%$ and specificity of $69.0 \%$. Cut off point for MAXH was set at 59.8 with sensitivity and specificity of $78.0 \%$ and $60.0 \%$, respectively. For BH, 49.5 was set as the cut off point with a sensitivity of $79.0 \%$ and specificity of $64.0 \%$. The best cut off point for CFH was $2: 7.8$ with $76.0 \%$ sensitivity and $63.0 \%$ specificity.

Conclusion: It can be concluded that the calcaneal diameters are reliable criteria for sex determination. Although the cutoff points are different between various races and populations, it is evident that these diameters can be used for sex determination in general.

Keywords: Gender determination, X-ray, Calcaneus, Iranian population

Conflicts of Interest: None declared

Funding: None

*This work has been published under CC BY-NC-SA 1.0 license.

Copyright $($ Iran University of Medical Sciences

Cite this article as: Faress F, Ameri M, Azizi H, Saboori Shekofte H, Hosseini R. Gender determination in adults using calcaneal diameters from lateral foot X-ray images in the Iranian population. Med J Islam Repub Iran. 2021 (14 Jun);35:76. https://doi.org/10.47176/mjiri.35.76

\section{Introduction}

Nowadays, one of the trending topics in forensic an-

thropology and archeology is determining the unknown

Corresponding author:Dr Maryam Ameri, ameri.m@iums.ir

1. Department of Legal Medicine \& Toxicology, Iran University of Medical Sciences, Tehran, Iran

2. Department of Internal medicine, Kosar Hospital, Semnan, Iran

3. Department of Nephrology, Iran University of Medical Sciences, Tehran, Iran $\uparrow$ What is "already known" in this topic:

Estimation of sex from human remains has considerable forensic value. The calcaneus is the largest foot bone, and its fractures are rare during postmortem changes. The mean values for calcaneal dimensions can be used for sex determination based on recent studies.

\section{$\rightarrow$ What this article adds:}

This study evaluated the calcaneal diameters in the Iranian population for the first time. Most of the studies in the field use human remains and skeletal libraries to gather the measurements manually. But in this study, we used computerized X-ray images from the live population which is more accurate than manual measurements. It can be concluded that the calcaneal diameters are reliable criteria for sex determination in the Iranian population. Although the cutoff points are different between various races and populations, it is evident that these diameters can be used for sex determination in general. 
sex from the evaluation of the bone remains (1). In some cases, sex identification is not possible even from the soft tissue present in the remains, which leave the skeletal system as the only evidence in gender differentiation (2-4). Determining gender is one of the main challenges in forensic medicine (5).

Evaluation of sex differences is vastly diverse in various races and nationalities. It is obligatory for each nation to understand the skeletal and tissue differences between the two sexes. Surely sex determination using these techniques is more convenient, faster, and cheaper than molecular evaluation such as evaluation of genetics and chromosomes $(6,7)$.

There are two general methods in gender determination from the skeletal system: the morphologic and the metric methods. The morphologic approach is quick and experimental, which requires specialized training and a lot of experience in this field. In the metric method, gender is determined by measuring bone diametric indexes which has higher reliability (8-10). For this approach, various indexes have been evaluated and set such as the shape of the bone, height, length, width and etc. Many bones are used to determine the gender including skull, sternum, hand, foot, pelvis and even teeth $(11,12)$.

Calcaneus is the largest bone in the foot, which is usually well preserved in skeletal remains due to the covers such as socks or shoes. Therefore this bone can be useful in gender determination from the body remains (13).

Almost every bone in the body is used in gender determination in forensic medicine, but each bone has its own sensitivity. For calcaneus, previous studies have reported a sensitivity from 67 percent to 97 percent (14-16).

According to literature, 11 indexes have been studied before for calcaneus: Boehler angle, maximum anteriorposterior length, maximum height, cuboid facet height, height of the calcaneal body, length of the loading arm, minimum width, maximum width, dorsal articular facet surface, dorsal articular facet length and width of the sulcus canal (17-19).

To our knowledge, there hasn't been a study reviewing the gender determination from calcaneus bone in the Iranian population. This study evaluated the calcaneal diameters in the Iranian population for the first time. Most of the studies in the field use human remains and skeletal libraries to gather the measurements manually. But in this study, we use computerized X-ray images from the live population which is more accurate than manual measurements. Since it is necessary for each nation to know its own indexes for gender determination, we studied the calcaneus bone between the two sexes.

\section{Methods}

This cross-sectional study was conducted on Iranians referring to Hazrat-e Rasool Hospital's radiology ward for plain lateral X-ray of the foot. 200 patients aged between 18 and 80 years old who did not suffer major trauma to the calcaneus bone were recruited after gathering informed consent. Also, patients with a history of surgeries on the calcaneus bone and congenital abnormalities of the foot were not included. This study was approved by the ethics committee of Iran University of medical sciences.

$\mathrm{X}$-rays were obtained in stanidard lateral view of the foot by an experienced technician. Using a picture archiving and communication system (PACS), four variables were calculated for each X-ray graph:

1. Maximum anterior-posterior length of the calcaneus bone (MAXL): the distance between the most anterior part of the calcaneus and the most posterior part on the calcaneus tuberosity.

2. Maximum height of the bone (MAXH): the length of the most superior and the most inferior part of the calcaneal tuberosity.

3 . Height of the cuboid facet $(\mathrm{CFH})$ : the distance between the most superior and the most inferior part of the articular facet for the cuboid.

4. Height the calcaneal body (BH): the distance between the superior and inferior surfaces of the calcaneal body in the coronal plane which was measured at the midpoint between the most anterior point and the most inferior point of the bone.

All measurements were linear distances which were measured by an expert technician, with the knowledge of the foot anatomy, using the PACS software. A second expert confirmed the bone measurements by re-measuring all the measurements. In the cases with different measurements, mean of the measurements was used.

For statistical analysis, Statistical Package for Social Sciences, version 20.0 (IBM SPSS Statistics Inc., Chicago, IL, USA) was used. Quantitative variables were described using means and standard deviation (SD). For qualitative data, frequency and percentages were used. The receiver operating characteristics (ROC) curve was employed to compare different bone features between the two sexes. $\mathrm{P}<0.05$ was considered as the level of significance.

\section{Results}

Lateral foot X-rays from 100 men and 100 women were gathered and evaluated for callcaneal indexes. The means for the four indexes are detailed in Table 1. These means were compared between the sexes, which showed all four means are different significantlly.

Also, five ratio indexes (MAXL to MAXH, MAXL to $\mathrm{BH}, \mathrm{MAXL}$ to $\mathrm{CFH}, \mathrm{MAXH}$ to $\mathrm{BH}$ and $\mathrm{MAXH}$ to $\mathrm{CFH}$ )

\begin{tabular}{lccc}
\multicolumn{4}{l}{ Table 1. Means for calcaneal indexesi } \\
\hline Index & Men & Women & p \\
\hline MAXL & $92.76 \pm 6.69$ & $84.74 \pm 5.36$ & $<0.001$ \\
MAXH & $64.69 \pm 6.46$ & $58.88 \pm 4.67$ & $<0.001$ \\
BH & $53.07 \pm 4.79$ & $48.27 \pm 4.34$ & $<0.001$ \\
CFH & $30.29 \pm 3.17$ & $27.04 \pm 3.00$ & $<0.001$ \\
\hline
\end{tabular}

\begin{tabular}{lccc}
\multicolumn{4}{l}{ Table 2. Comparison of ratio indices between man and women } \\
\hline Index & Men & Women & $\mathrm{p}$ \\
\hline MAXL to MAXH & $1.44 \pm 0.11$ & $1.44 \pm 0.11$ & 0.838 \\
MAXL to BH & $1.76 \pm 0.13$ & $1.76 \pm 0.17$ & 0.810 \\
MAXL to CFH & $3.08 \pm 0.29$ & $3.16 \pm 0.33$ & 0.069 \\
MAXH to BH & $1.23 \pm 0.14$ & $1.23 \pm 0.13$ & 0.936 \\
MAXH to CFH & $2.14 \pm 0.22$ & $2.20 \pm 0.26$ & 0.132 \\
\hline
\end{tabular}


were calculated which neither showed a significant difference between men and women. The comparison for these ratios is described in Table 2.

Cut off points for each index were calculated using the area under curve (AUC) in ROC curves. AUC for MAXL in differentiating the genders was 0.824 which showed 86.8 as the cut off with sensitivity of $80.0 \%$ and specificity of $69.0 \%$. Cut off point for MAXH was set at 59.8 with sensitivity and specificity of $78.0 \%$ and $60.0 \%$, respectively. For BH, 49.5 was set as the cut off point with sensitivity of $79.0 \%$ and specificity of $64.0 \%$. The best cut off point for $\mathrm{CFH}$ was 27.8 with $76.0 \%$ sensitivity and $63.0 \%$ specificity. The ROC curves are shown in Figures 1-4.

Also, the ROC curve was used for ratio indexes which revealed that neither are able to differentiate between the genders (Table 3).

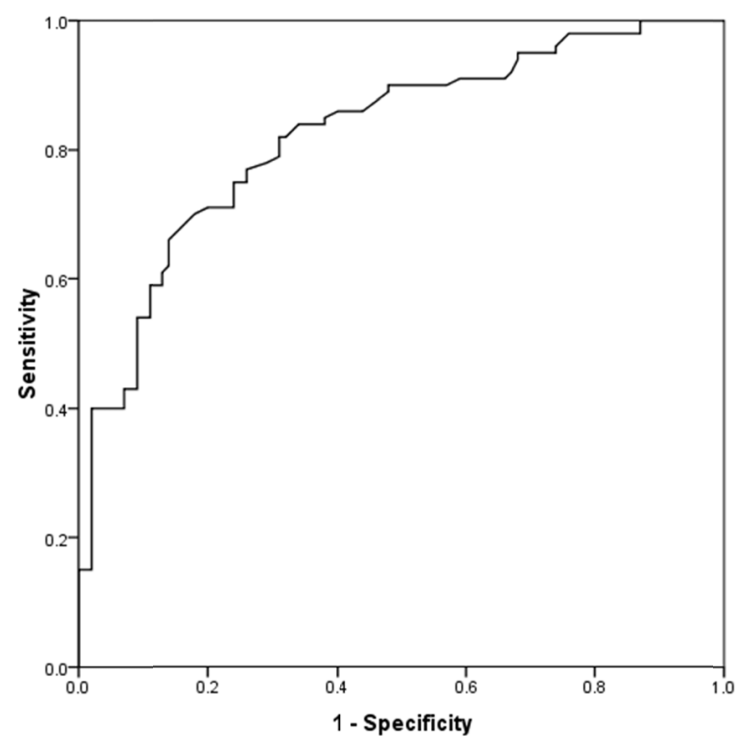

Fig. 1. The ROC curve for MAXL

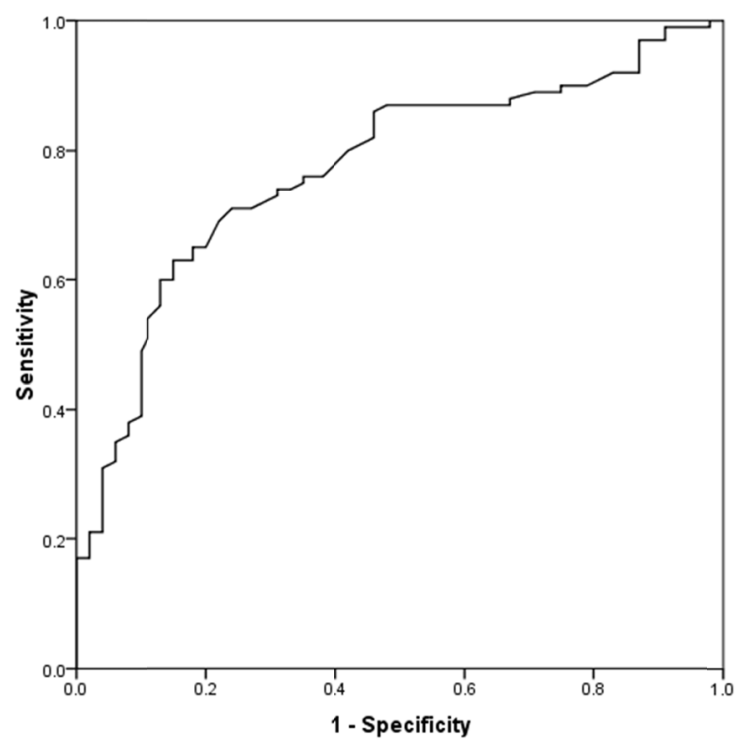

Fig. 2. The ROC curve for MAXH

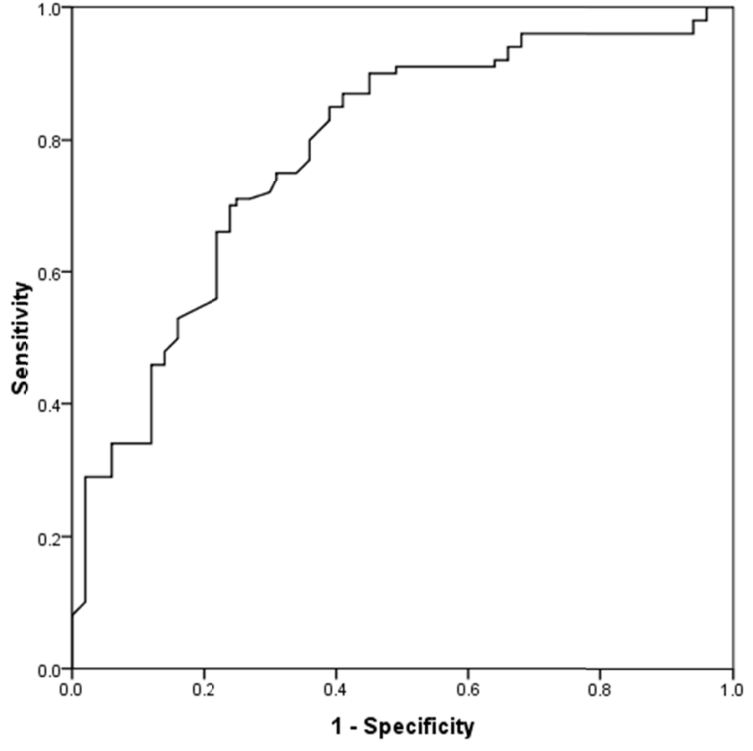

Fig. 3. The ROC curve for BH

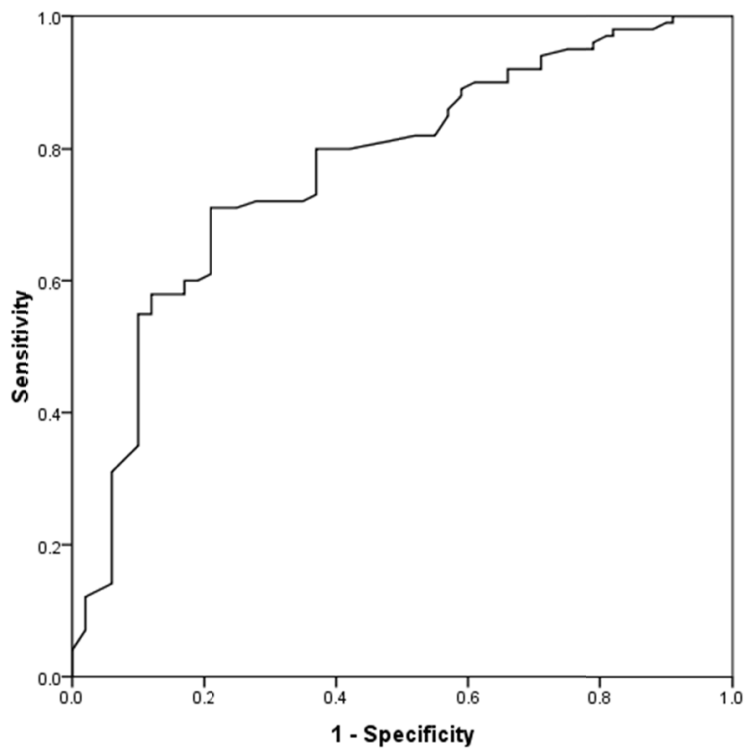

Fig. 4. The ROC curve for $\mathrm{CFH}$

Table 3. ROC evaluation for ratio indices

\begin{tabular}{lccc}
\hline Index & AUC & CI & p \\
\hline MAXL to MAXH & 0.497 & $0.417-0.578$ & 0.949 \\
MAXL to BH & 0.489 & $0.408-0.571$ & 0.796 \\
MAXL to CFH & 0.421 & $0.341-0.501$ & 0.996 \\
MAXH to BH & 0.484 & $0.403-0.565$ & 0.699 \\
MAXH to CFH & 0.439 & $0.359-0.519$ & 0.135 \\
\hline
\end{tabular}

\section{Discussion}

Using morphologic features of the bones is the basis of gender determination in anthropology and forensic medicine. For this purpose, each of the bone diameters has accuracy in determining the gender which is different between various races (20). Foot bones are sexually dimorphic, and they remain good after postmortem damages so they are suitable for sex identification. In this study, we evaluated the calcaneus diameters for gender determina- 
tion in the Iranian population.

We reported that all four diameters of MAXL, MAXH, $\mathrm{BH}$, and $\mathrm{CFH}$ were significantly different between the two genders; therefore they can be used in gender determination in the Iranian population. Also, by analyzing the area under the ROC curve, acceptable specificity and sensitivity were obtained for each of the diameters.

Scott et al. reviewed nine calcaneal measurements in the Thai population and reported that all nine measurements were significantly different between the genders, which was similar to our study (14). Scott reported higher accuracy of sex determination for MAXL, MAXH, BH and CFH than the numbers that we have achieved. Scott used the skeletons to measure calcaneal indexes manually but in this study, we used radiologic images and computerized measuring which yields a higher accuracy which might be the cause of the difference seen between the two studies.

Peckmann et al. studied the same 9 measurements in the Greek population. He also reported that all nine indexes had significant differences between the sexes. The cut off points calculated by Peckmann were lower than our cutoff points which is predictable because of the racial differences. This difference shows that skeletal measurements are highly population specific (13).

Kim et al. evaluated 10 markers on the calcaneus for sex differentiation in the Korean population (21). Kim's results were aligned with our results. In his study, the MAXL and minimum breadth of the bone had the highest accuracy for sex determination. Our study also showed the MAXL as the most reliable diameter for sex determination. Bidmos et al. measurements showed that the MAXL is more different between the genders and has a higher accuracy for gender determination (22).

Zakaria et al. studied the calcaneal diameters and angles use for gender determination in Egyptians (15). He used $\mathrm{X}$-ray images of the foot to calculate the angles and length as we did in this study. The study showed the same results that the calcaneal diameters are reliable in sex determination but he reported that calcaneal angles are not significantly different between the sexes.

This study evaluated the calcaneal diameters in the Iranian population for the first time. Most of the studies in the field use human remains and skeletal libraries to gather the measurements manually. But in this study, we used computerized X-ray images from the live population which is more accurate than manual measurements.

\section{Conclusion}

It can be concluded that the calcaneal diameters are reliable criteria for sex determination. Although the cutoff points are different between various races and populations, it is evident that these diameters can be used for sex determination in general.

\section{Acknowledgment}

The authors thank Hazrat-e Rasool Hospital staffs for their cooperation in this research.

\section{Conflict of Interests}

The authors declare that they have no competing interests.

\section{References}

1. Krishan K, Kanchan T, Asha N, Kaur S, Chatterjee P, Singh B. Estimation of sex from index and ring finger in a North Indian population. J Forensic Leg Med. 20113;20:471-9.

2. Khanpetch P, Prasitwattanseree S, Case DT, Mahakkanukrauh P. Determination of sex from the metacarpals in a Thai population. Forensic Sci Int. 2012;217(1-3):229.e1-8.

3.Zech WD, Hatch G, Siegenthaler L, Thali MJ, Losch S. Sex determination from os sacrum by postmortem CT. Forensic Sci Int. 2012;221(1-3):39-43.FSI

4. Kanchan T, Krishan K. Anthropometry of hand in sex determination of dismembered remains - A review of literature. J Forensic Leg Med. 2011;18(1):14-7.

5. Mostafa E, El-Elemi A, El-Beblawy M, Dawood A. Adult sex identification using digital radiographs of the proximal epiphysis of the femur at Suez Canal University Hospital in Ismailia, Egypt. Egypt J Forensic Sci. 2012;2:81-8.

6. Ahmed AA. Estimation of sex fronn the upper limb measurements of Sudanese adults. J Forensic Leg Med. 2013;20(8):1041-7.

7. Charisi D, Eliopoulos C, Vanna V, Koilias CG, Manolis SK. Sexual dimorphism of the arm bones in a modern greek population. J Forensic Sci. 2011;56(1):10-8.

8. Mitra A, Khadijeh B, Vida AP, Ali RN, Farzaneh M, Maryam VF, et al. Sexing based on measurements of the femoral head parameters on pelvic radiographs. J Forensic Leg Med. 2014;23:70-5.

9. Gonzalez PN, Bernal V, Perez SI. Geometric morphometric approach to sex estimation of human pelvis. Forensic Sci Int. 2009;189(1-3):6874.

10. Hayashizaki Y, Usui A, Hosokai Y, Sakai J, Funayama M. Sex determination of the pelvis using Fourier analysis of postmortem CT images. Forensic Sci Int. 2015;246:122.e1-9.FSI

11. Jowaheer V, Agnihotri AK. Sex identification on the basis of hand and foot measurements in Indo-Mauritian population--a model based approach. J Forensic Leg Med. 20111;18(4):173-6.

12. Clavero A, Salicru M, Turbon D. Sex prediction from the femur and hip bone using a sample of CT images from a Spanish population. Int J Leg Med. 2015;129(2):373-83.

13. Peckmann TR, Orr K, Meek S, Manolis SK. Sex determination from the calcaneus in a 20th century Greek population using discriminant function analysis. Sci Justice. 2015;55(6):377-82.

14. Scott S, Ruengdit S, Peckmann TR, Mahakkanukrauh P. Sex estimation from measurements of the calcaneus: Applications for personal identification in Thailand. Forensic Sci Int. 2017;278:405.e1.e8.

15. Zakaria MS, Mohammed AH, Habib SR, Hanna MM, Fahiem AL. Calcaneus radiograph as a diagnostic tool for sexual dimorphism in Egyptians. J Forensic Leg Med. 201 0;17(7):378-82.

16. Nathena D, Michopoulou E, Kranioti EF. Sexual dimorphism of the calcaneus in contemporary Cretans. Forensic Sci Int. 2017;277:260.e1-.e8.

17. Ekizoglu O, Inci E, Palabiyik FBi, Can IO, Er A, Bozdag M, et al. Sex estimation in a contemporary Turkish population based on CT scans of the calcaneus. Forensic Sci Int. 2017;279:310.e1-.e6.

18. Gualdi-Russo E. Sex determination from the talus and calcaneus measurements. Forensic Sci Int. 2007; 171(2-3):151-6.

19. Bidmos MA, Dayal MR. Sex determination from the talus of South african whites by discriminant function analysis. AM J Forensic Med Pathol. 2003;24(4):322-8.

20. Introna F Jr, Di Vella G, Campobasso CP, Dragone M. Sex determination by discriminant anallysis of calcanei measurements. J Forensic Sci. 1997;42(4):725-8.

21. Kim DI, Kim YS, Lee UY, Han SH. Sex determination from calcaneus in Korean using discrimninant analysis. Forensic Sci Int. 2013;228(1-3):177.e1-7.

22. Bidmos MA, Asala SA. Sexual dimorphism of the calcaneus of South African blacks. J Forensic Scii. 2004;49(3):446-50. 